\title{
White blood cell count and cardiovascular biomarkers of atherosclerosis
}

YUI SEKITANI*, NAOMI HAYASHIDA*, KOICHIRO KADOTA ${ }^{\dagger}$, HIRONORI YAMASAKI ${ }^{\ddagger}$ NORIO ABIRU', MIO NAKAZATO ${ }^{\#}$, TAKAHIRO MAEDA YOSHIYUKI OZONO ${ }^{\dagger}$ and NOBORU TAKAMURA*

*Department of Radiation Epidemiology, Nagasaki University Graduate School of Biomedical Sciences, Nagasaki, Japan

${ }^{\dagger}$ Department of General Medicine, Nagasaki University Graduate School of Biomedical Sciences, Nagasaki, Japan

Center for Health and Community Medicine, Nagasaki University, Nagasaki, Japan "Department of Endocrinology and Metabolism, Unit of Translational Medicine, Nagasaki University Graduate School of Biomedical Sciences, Nagasaki, Japan \#Department of Island and Community Medicine, Nagasaki University Graduate School of Biomedical Sciences, Nagasaki, Japan

Correspondence to:

Noboru Takamura, M.D., Ph.D.

Professor, Department of Radiation Epidemiology

Nagasaki University Graduate School of Biomedical Sciences

1-12-4 Sakamoto, Nagasaki 8528523, Japan

Tel: +81-95-819-7170; Fax: +81-95-819-7172

E-mail: takamura@nagasaki-u.ac.jp 
Key words

Cardio-ankle vascular index, Carotid intima-media thickness, Metabolic syndrome 


\begin{abstract}
Objective: To investigate the association with white blood cell (WBC) and atherosclerotic parameters including cardio-ankle vascular index (CAVI) and carotid intima-media thickness (CIMT) in the general population.
\end{abstract}

Methods: We investigated the relationship between WBC count and metabolic syndrome components, CAVI, CIMT in 3738 Japanese study participants.

Results: WBC count weakly correlated with CAVI in men $(\beta=0.61, p=0.043)$, but not in women $(\beta=0.35, p=0.17)$. On the other hand, WBC did not correlate with CIMT in either men or women ( $p=0.41$ and $p=0.71$, respectively).

Conclusion: WBC count was associated with lipids, blood pressure and body mass index, although the correlations with CAVI and CIMT were weak or absent. 


\section{Introduction}

White blood cells (WBC) comprise an inexpensive and reliable marker of inflammation that is universally applied in standard clinical practice. Recently atherosclerosis has been recognized as an active, inflammatory process (Ross 1999), and has been discussed whether the elevation of WBC is merely a marker of inflammation, whether it directly enhances atherosclerosis and thrombosis, or both. It is still remained unclear whether a relationship between WBC and subclinical atherosclerosis exists. Several studies have shown a positive and independent association between WBC and specific diseases including cancer (Grimm et al. 1985 and Friedman et al. 1991), cardiovascular disease (CVD) (Grimm et al. 1985 and Prentice et al. 1982), stroke (Prentice et al. 1982) and all-cause mortality (Grimm et al. 1985). Furthermore, Lao et al. recently identified a strong relationship between total WBC count and the constellation of metabolic syndrome components in an older Chinese population (Lao et al. 2008). Therefore, detecting a high WBC count or a change in the count might be important in predicting subsequent disease.

Recent advances in medical technology have allowed noninvasive assessment of early atherosclerosis (Vogel et al. 2000 and Bots et al. 2002). High-resolution B-mode 
ultrasonography provides a noninvasive method of measuring arterial wall thickening, and indeed, this modality has shown that carotid intima-media thickness (CIMT) is a powerful predictor of CVD (Bots et al. 2002), as it correlates with generalized atherosclerosis, myocardial infarction and stroke (Bots et al. 1997). Population studies have revealed that traditional cardiovascular risk factors such as age, smoking, low-density lipoprotein-cholesterol (LDL-C) and elevated blood pressure (BP) are closely associated with CIMT (Loimaala et al. 2006).

Pulse wave velocity (PWV) is a noninvasive clinical index of aortic stiffness (van Popele et al. 2001), and it can predict cardiovascular events as well as all-cause mortality in hypertensive patients and in the general population (Blacher et al. 1999 and Shokawa et al. 2005). The novel cardio-ankle vascular index (CAVI), which reflects the stiffness of the aorta, femoral artery and tibial artery, involves the measurement of brachial-ankle PWV and BP (Yambe et al. 2004 and Shirai et al. 2006). This index is essentially independent of changes in BP during the examination, but closely correlates with systolic BP. Kadota et al. suggested that CAVI, like PWV, can serve as a marker of the increase in central artery stiffness with age (Kadota et al. 2008). Although Wakabayashi et al. revealed a correlation between WBC and CAVI in patients with type 2 diabetes mellitus (Wakabayashi et al. 2006), this aspect has not been investigated in 
the general population.

In this present study, we investigated the association with WBC and atherosclerotic parameters including CAVI and CIMT in a relatively large general population. 


\section{Methods}

The Special Committee of Nagasaki University provided ethical approval to proceed with this study (project registration number 0501120073), during a medical screening program for the general population over 20 years old, residing in Goto City (total population, 41,729 in 2008), Nagasaki Prefecture, Japan between 2005 and 2009. Data were collected by the staff of Nagasaki University, in cooperation with the staff of Goto city. We obtained written, informed consent from 4992 Japanese adults (1640 men and 3352 women) to participate in the study. However, 51 of them with severe hypertriglycemia $(>4.0 \mathrm{~g} / \mathrm{l})$ and 1203 in whom WBC, CAVI and CIMT were not measured were excluded from the study. Data from a total of 3738 participants (1165 men and 2573 women) were finally included for further analysis.

The height and weight of each participant were measured and body mass index (BMI: kilograms per meter square) was calculated as an index of obesity. Waist circumference (WC) was measured horizontally at the umbilicus with a tape measure after normal expiration. Systolic blood pressure (SBP) and diastolic blood pressure (DBP) were recorded at rest. Mean blood pressure $(\mathrm{MBP})$ was calculated as DBP + (SBP-DBP)/3.

Blood samples were collected from each participant after an overnight fast. Serum 
and plasma were separated and stored at $-20^{\circ} \mathrm{C}$ and $-80^{\circ} \mathrm{C}$, respectively, until assay. Both white (WBC) and red (RBC) blood cells were counted, and hemoglobin (HGB) and hematocrit (HCT) were measured using standard laboratory procedures. Serum concentrations of total cholesterol (TC), triglyceride (TG), and high-density lipoprotein-cholesterol (HDL-C) were also measured using standard laboratory procedures and LDL-C was calculated using the Friedewald equation (Friedewald et al. 1972). In addition, hemoglobin $A_{1 c}\left(H_{b A}\right)$, serum creatinine (CRE) and uremic acid (UA) were measured using standard laboratory procedures.

The physicians (Y.S., N.H., M.N. and N.T.) measured CIMT by ultrasonography of the right and left carotid arteries using a LOGIC Book XP with a 10-MHz linear array transducer (GE Medical Systems, Milwaukee, WI, USA). The far wall of the carotid artery was displayed on a longitudinal two-dimensional ultrasonographic image as two bright white lines separated by a hypoechoic space. The distance from the leading edge of the first (lumen-intima interface), to the leading edge of the second (media-adventitia interface) bright line was identified as the CIMT. Images obtained were stored on the hard disk of the ultrasound system, and were analyzed using Intima Scope ${ }^{\circledR}$ software (MEDIA CROSS, Tokyo, Japan). Averages of the right and left CIMT were calculated and used in the analysis. Intra- (N.T., $n=32)$ and inter- (N.T. vs. M.N., $n=41)$ observer 
variations in CIMT were $0.91(p<0.01)$ and $0.78(p<0.01)$, respectively.

The CAVI in supine participants was recorded using a VaseraVS-1000 vascular screening system (Fukuda Denshi, Tokyo, Japan). The principles underlying CAVI have been described by Yambe et al. (Yambe et al. 2004). Electrocardiographic electrodes are placed on both wrists, a microphone for detecting heart sounds is placed on the sternum, and cuffs are wrapped around both arms and ankles to obtain automatic measurements. The data were then analyzed using VSS-10 software (Fukuda Denshi), and calculated averaged values for the right and left CAVI further analyzed.

Results are expressed as means \pm standard deviation or median $\left(25^{\text {th }}-75^{\text {th }}\right.$ quartile $)$. Differences in laboratory values between men and women were evaluated using a $t$-test and the Mann-Whitney U-test. Smoking status was analyzed among three groups; i.e. current smoker, past smoker and never smoked. Differences in the smoking status and the ratio of current treatment for hypertension (HT), diabetes mellitus (DM) and dyslipidemia (DL) were evaluated using the $\chi^{2}$ test. White blood cells and other variables including laboratory values, CAVI and CIMT were evaluated using univariate linear regression analysis. Multivariate linear regression analysis was adjusted for age to evaluate WBC and other existing parameters. We evaluated associations between WBC and CAVI, CIMT using another multivariate linear regression analysis adjusted for age 
and other confounding factors such as BMI, MBP, $\mathrm{HbA}_{1 \mathrm{c}} \mathrm{HDL}-\mathrm{C}$. To confirm whether relationships between WBC and CAVI, CIMT are independent of circulation dynamics, lipid metabolism and glucose metabolism, multivariate linear regression analysis was adjusted for age, BMI, MBP, $\mathrm{HbA}_{1 \mathrm{c}}$, HDL-C and current treatment for $\mathrm{HT}$ and/or DM and/or DL. Because the distribution of TG was skewed, logarithmic transformation was performed for the univariate and multivariate linear regression analyses. Probability values below 0.05 were considered indicative of statistical significance. All data were statistically analyzed using SPSS v17.0 software (SPSS Japan, Tokyo, Japan). 


\section{Results}

Table 1 shows the characteristics of the study participants. The men were significantly older than the women (average: $66.0 \pm 10.9$ vs. $64.0 \pm 11.2$ years, $p<0.01$ ). Besides age, BMI, WC, MBP, WBC, RBC, HGB, HCT, CRE, UA, TC, TG, HDL-C, LDL-C, CAVI and CIMT, as well as the ratio of current smoker, past smoker and current treatment for DM and DL, significantly differed between men and women. The number of participants who are diagnosed as HT, DM and DL though not under drug treatment were 12, 24 and 31 respectively in the whole participants.

Univariate linear regression analysis showed that WBC correlated in men and in women with age, BMI, WC, MBP, RBC, HGB, HCT, UA, TC, log TG, HDL-C, LDL-C and $\mathrm{HbA}_{1 \mathrm{c}}$. In addition, smoking status in men, and CRE in women also correlated (Table 2). This analysis revealed that WBC did not correlate with CAVI and CIMT in either men or women.

Multivariate linear regression analysis adjusted for age revealed that WBC correlated with BMI, WC, smoking status, MBP, RBC, HGB, HCT, CRE, UA, TC, log TG, HDL-C, LDL-C, HbA $1 \mathrm{c}, \mathrm{CAVI}$ and CIMT in men. In women, WBC correlated with the same parameters except for smoking status, CAVI and CIMT (Table 3). 
Multivariate linear regression analysis adjusted for confounding factors including CAVI showed that WBC correlated with age $(\beta=-0.19, p<0.001)$, BMI $(\beta=0.64, p<$ $0.001)$, smoking status $(\beta=5.1, p<0.001)$, and HDL-C $(\beta=-0.093, p=0.002)$ in men, and with age $(\beta=-0.18, p<0.001)$, BMI $(\beta=0.33, p<0.001)$, MBP $(\beta=0.049, p=$ $0.025), \mathrm{HbA}_{1 \mathrm{c}}(\beta=1.1, p=0.017)$ and HDL-C $(\beta=-0.075, p<0.001)$ in women (Table 4). The WBC count weakly correlated with CAVI in men $(\beta=0.61, p=0.043)$ though not in women $(\beta=0.35, p=0.17)$. In the whole participants, WBC correlated in all confounding factors and also with CAVI $(\beta=0.4, p=0.028)$. Furthermore, multivariate linear regression analysis adjusted for confounding factors including current treatment for $\mathrm{HT}$ and/or DM and/or DL showed that WBC did not correlate with CAVI in men, women and all ( $p=0.059, p=0.27$ and $p=0.063$, respectively; data not shown).

Multivariate linear regression analysis adjusted for confounding factors including CIMT showed that WBC correlated with age $(\beta=-0.17, p<0.001)$, BMI $(\beta=0.62, p<$ $0.001)$, smoking status $(\beta=5.1, p<0.001), \operatorname{MBP}(\beta=0.072, p=0.036)$, and HDL-C $(\beta=-0.094, p=0.002)$ in men, and with age $(\beta=-0.16, p<0.001), \operatorname{BMI}(\beta=0.32, p<$ $0.001), \operatorname{MBP}(\beta=0.052, p=0.019), \operatorname{HbA}_{1 \mathrm{c}}(\beta=1.2, p=0.013)$, and HDL-C $(\beta=-0.075$, $p<0.001)$ in women. WBC did not correlate with CIMT in either men or women $(p=$ 0.41 and $p=0.71$, respectively; Table 5). In the whole participants, WBC correlated in 
all confounding factors, although it did not correlate with CIMT $(\beta=1.2, p=0.51)$.

Multivariate linear regression analysis adjusted for confounding factors including current treatment for HT and/or DM and/or DL also showed that WBC did not correlate with CIMT in men, women and all $(p=0.46, p=0.79$ and $p=0.58$, respectively; data not shown). 


\section{Discussion}

In this study, we showed that the WBC count weakly correlated with CAVI in men but not in women and that it did not correlate with CIMT in either gender after adjustment for confounding factors. To our knowledge, this is the first analysis of the relationship between $\mathrm{WBC}$ and atherosclerosis represented by CIMT and CAVI in a large community-based sample. We also showed that WBC was strongly correlated with metabolic syndrome markers such as BMI, WC, MBP, HbA 1 , TC, LDL-C and HDL-C, which is consistent with the findings of Lao et al. (Lao et al. 2008). Their report also showed that the prevalence of metabolic syndrome significantly increased with higher WBC counts in both genders.

Our results showed a positive association between $\mathrm{BMI}$ and $\mathrm{WBC}$, which is also consistent with previous findings (Saito et al. 2003). Obesity plays an important role in the pathogenesis of atherosclerosis through low-grade, chronic inflammation. Elevated free fatty acids associated with obesity, insulin resistance, DM and metabolic syndrome cause endothelial dysfunction by activating innate immune inflammatory pathways upstream of the nuclear transcription factor, nuclear factor-kappaB (NF-kB) (Vincent et al. 2003), which promotes the synthesis and release of pro-inflammatory cytokines that 
enhance the attachment of monocytes and macrophages to the vessel walls [21]. Damage to the vessel walls causes endothelium and smooth muscle cells to become transcriptionally active and synthesize pro-inflammatory proteins, including chemokines, cell adhesion molecules and cytokines.

Smoking is known as a risk factor for atherosclerosis. In our study, WBC associated with smoking status in men though not in women. This is probably due to the small number of smokers in women. Previous studies showed that there is a strong, positive relationship between the number of cigarettes smoked and WBC count (Hansen et al. 1990 and Petitti et al. 1986).

Shankar et al. showed that the WBC count is associated with incidental hypertension in both genders independently of smoking and most traditional cardiovascular risk factors in a predominantly Caucasian cohort (Shankar et al. 2004). We found here that WBC also correlated with blood pressure in Japanese subjects. The concentration of the vasoconstrictor angiotensin II is often elevated in hypertensive patients. Angiotensin II can also contribute to atherogenesis by stimulating the growth of smooth muscle and its lipoxygenase activity, which can increase inflammation and LDL-C oxidation (Ross 1999).

The WBC count significantly correlates with $\mathrm{HbA}_{1 \mathrm{c}}$ in both $\mathrm{DM}$ and non-DM 
patients with known coronary atherosclerosis, and this indicates an early association between glycemia, inflammation and atherosclerosis before DM develops (Gustavsson et al. 2004). We also found a correlation between $\mathrm{WBC}$ and $\mathrm{HbA}_{1 \mathrm{c}}$, which suggests that low-grade chronic inflammatory activity increases in hyperglycemia. One possible explanation for this is that both a higher WBC count and insulin resistance reflects underlying activation of the immune system. Diabetes mellitus is considered to be associated with activation of the innate immune system, and acute-phase reactants, such as C-reactive protein and sialic acid, are proposed predictors of the risk of developing type 2 DM (Crook 2004). A chronic inflammatory response involved in the atherosclerosis process might increase blood concentrations of acute-phase reactants including WBC. Cytokines such as interleukin-6, which is a potent WBC differentiation factor, are associated with insulin resistance (Fernandez-Real et al. 1997). Activation of the immune system caused by inflammation could increase WBC, and cytokine production might therefore decrease insulin sensitivity.

We found that TG and LDL-C were positively associated with $\mathrm{WBC}$ and that HDL-C was inversely associated. Wilson et al. identified a relationship between lipoprotein cholesterol levels and WBC in the Framingham heart study (Wilson et al. 1983); an elevated WBC count was associated with decreased HDL-C and increased 
LDL-C in both smokers and non-smokers, suggesting that a higher WBC count is related to a more atherogenic lipid factor. A major cause of damage to the endothelium and underlying smooth muscle is LDL-C. Particles of LDL-C trapped in an artery undergo progressive oxidation and engulfment by macrophages through scavenger receptors on the surfaces of the endothelium and smooth muscle cells. Oxidized LDL-C is a feature of atherosclerotic lesions.

White blood cells play a crucial role in initiating and propagating the atherosclerotic process (Shankar et al. 2004). Stimulated WBC have an increased tendency to adhere to the vascular endothelium and easily penetrate the intima, causing capillary leukocytosis and increased vascular resistance. Additionally, WBC release various hydrolytic enzymes, cytokines and growth factors that can induce further vascular damage (Ross 1999). This cascade causes endothelial dysfunction and alters arterial elastic properties, leading to structural stiffness.

Lee et al. reported a positive association between WBC and baPWV that was independent of classical cardiovascular risk factors (Lee et al. 2009). On the other hand, we showed a weak correlation between WBC and CAVI in men $(\beta=0.61, p=0.043)$, but not in women $(\beta=0.35, p=0.17)$. This might be associated with the recognized sex difference in the mechanism and pathophysiology of atherosclerosis (Huang et al. 2001). 
Furthermore, we found that WBC did not correlate with CIMT in either men or women, which is inconsistent with previous studies (Loimaala et al. 2006 and Magyar et al. 2003). Magyer et al. showed that the WBC count was significantly higher in patients with at least $30 \%$ internal carotid artery stenosis or occlusion than in controls, and that CIMT was increased in such patients compared with controls. In addition, WBC still correlated with CIMT after adjusting for lipid factors (Magyar et al. 2003). This contradiction might be due to the background of the study population and the method of statistical analyses. Further studies are needed to clarify the association between WBC and atherosclerosis represented by CAVI and CIMT.

Our study has some limitations. First, only one WBC measurement was included in the analysis, and whether an acute, brief inflammatory episode or chronic inflammation was responsible for the observed correlation could not be discriminated. We could not fully exclude individuals with relevant inflammation or infection, but although WBC varies from day to day (Pocock et al. 1989), a single measurement can nevertheless predict the risk of death and of specific diseases, including cancer and CVD (Hoffman et al. 2004 and Madjid et al. 2004). Secondly, we did not estimate the data of other acute inflammatory markers such as CRP and fibrinogen. Thirdly, we did not analyze by comparing participants with or without current treatments due to the relatively small 
number of participants who are not under drug treatment. At last, we only evaluated the whole WBC count and not the difference between the subtypes. 


\section{Conclusion}

We showed that the WBC count is strongly associated with metabolic syndrome components such as lipids, blood pressure and BMI, whereas correlations with CAVI and CIMT were weak or absent. Further evaluation is needed to clarify the value of the WBC count as a marker of atherosclerosis. 


\section{Declaration of interest}

This study was supported by a Grant-in-Aid from the Japan Society for the Promotion of Science (No.21590699) and the Ministry of Education, Culture, Sports, Science and Technology of Japan through the Nagasaki University Global COE program. All authors have no conflict of interests. 


\section{References}

Blacher J, Asmar R, Djane S, London GM, Safar ME. (1999). Aortic pulse wave velocity as a marker of cardiovascular risk in hypertensive patients. Hypertension $33: 1111-7$

Bots ML, Grobbee DE. (2002). Intima media thickness as a surrogate marker for generalized atherosclerosis. Cardiovasc Drugs Ther 16:341-51.

Bots ML, Hoes AW, Koudstaal PJ, Hofman A, Grobbee DE. (1997). Common carotid intima-media thickness and risk of stroke and myocardial infarction: the Rotterdam study. Circulation 96:1432-7.

Crook M. (2004). Type 2 diabetes mellitus: a disease of the innate immune system? An update. Diabet Med 21:203-7.

Fernandez-Real JM, Vayreda M, Richart C, Gutierrez C, Broch M, Vendrell J, Ricart W. (1997). Circulating interleukin 6 levels, blood pressure, and insulin sensitivity in apparently healthy men and women. J Clin Endocrinol Metab 82:4196-200.

Friedewald WT, Levy RI, Fredrickson DS. (1972). Estimation of the concentration of low-density lipoprotein cholesterol in plasma, without use of the preparative ultracentrifuge. Clin Chem 18:499-502. 
Friedman GD, Fireman BH. (1991). The leukocyte count and cancer mortality. Am $J$ Epidemiol 133:376-80.

Grimm RH, Neaton JD, Ludwig W. (1985). Prognostic importance of the white blood cell count for coronary, cancer, and all-cause mortality. JAMA 254:1932-7.

Gustavsson CG, Agardh CD. (2004). Markers of inflammation in patients with coronary artery disease are also associated with glycosylated haemoglobin Alc within the normal range. Eur Heart J 25:2120-4.

Hansen LK, Grimm RH Jr, Neaton JD. (1990). The relationship of white blood cell count to other cardiovascular risk factors. Int J Epidemiol 19:881-8.

Hoffman M, Blum A, Baruch R, Kaplan E, Benjamin M. (2004). Leukocytes and coronary heart disease. Atherosclerosis 172:1-6

Huang ZS, Jeng JS, Wang CH, Yip PK, Wu TH, Lee TK. (2001). Correlations between peripheral differential leukocyte counts and carotid atherosclerosis in non-smokers. Atherosclerosis 158:431-6.

Kadota K, Takamura N, Aoyagi K, Yamasaki H, Usa T, Nakazato M, Maeda T, Wada M, Nakashima K, Abe K, Takeshima F, Ozono Y. (2008). Availability of cardio-ankle vascular index (CAVI) as a screening tool for atherosclerosis. Circ J 72:304-8.

Koh KK. (2002). Effects of estrogen on vascular wall: vasomotor function and 
inflammation. Cardiovasc Res 55:714-26.

Lao XQ, Thomas GN, Jiang C, Zhang W, Adab P, Lam TH Cheng KK. (2008). White blood cell count and the metabolic syndrome in older Chinese: The Guangzhou biobank cohort study. Atherosclerosis 201:418-24.

Lee YJ, Lee JW, Kim JK, Lee JH, Kim JH, Kwon KY, Lee HR, Lee DC, Shim JY. (2009). Elevated white blood cell count is associated with arterial stiffness. Nutr Metab Cardiovasc Dis 19:3-7.

Loimaala A, Rontu R, Vuoli I, Mercuri M, Lehtimäki T, Nenonen A, Bond MG. (2006) Blood leukocyte count is a risk factor for intima-media thickening and subclinical carotid atherosclerosis in middle-aged men. Atherosclerosis 188:363-9.

Madjid M, Awan I, Willerson JT, Casscells SW. (2004). Leukocyte count and coronary heart disease: implications for risk assessment. J Am Coll Cardiol 44:1945-56.

Magyar MT, Szikszai Z, Balla J, Valikovics A, Kappelmayer J, Imre S, Balla G, Jeney V, Csiba L, Bereczki D. (2003). Early-onset carotid atherosclerosis is associated with increased intima-media thickness and elevated serum levels of inflammatory markers. Stroke 34:58-63.

Petitti DB, Kipp H. (1986). The leukocyte count: associations with intensity of smoking and persistence of effect after quitting. Am J Epidemiol 123:89-95. 
Pocock SJ, Ashby D, Shaper AG, Walker M, Broughton PM. (1989). Diurnal variations in serum biochemical and hematological measurements. J Clin Pathol 42:172-9.

Pretince RL, Szatrowski TP, Fujikura T, Kato H, Mason MW, Hamilton HH. (1982). Leukocyte counts and coronary heart disease in a Japanese cohort. Am J Epidemiol 116:496-509.

Prentice RL, Szatrowski TP, Kato H, Mason MW. (1982). Leukocyte counts and cerebrovascular disease. J Chronic Dis 35:703-14.

Ross R. (1999). Atherosclerosis--an inflammatory disease. N Engl Med 340:115-26.

Saito I, Yonemasu K, Inami F. (2003). Association of body mass index, body fat, and weight gain with inflammation markers among rural residents in Japan. Circ J 67:323-9.

Shankar A, Klein BE, Klein R. (2004). Relationship between white blood cell count and incident hypertension. Am J Hypertens 17:233-9.

Shirai K, Utino J, Otsuka K, Takata M. (2006). A novel blood pressure-independent arterial wall stiffness parameter; cardio-ankle vascular index (CAVI). $J$ Atheroscler Thromb 13:101-7.

Shokawa T, Imazu M, Yamamoto H, Toyofuku M, Tasaki N, Okimoto T, Yamane K, Kohno N. (2005). Pulse wave velocity predicts cardiovascular mortality: Findings 
from the Hawaii-Los Angeles-Hiroshima study. Circ J 69:259-64.

van Popele NM, Grobbee DE, Bots ML, Asmar R, Topouchian J, Reneman RS, Hoeks APG, van der Kuip DAM, Hofman A, Witteman JCM. (2001). Association between arterial stiffness and atherosclerosis: the Rotterdam study. Stroke 32:454-60.

Vincent MA, Montagnani M, Quon MJ. (2003). Molecular and physiologic actions of insulin related to production of nitric oxide in vascular endothelium. Curr Diab Rep $3: 279-88$.

Vogel RA, Benitez MR. (2000). Noninvasive assessment of cardiovascular risk: From Framingham to the future. Rev Cardiovasc Med 1:34-42.

Wakabayashi I, Masuda H. (2006). Association of acute-phase reactants with arterial stiffness in patients with type 2 diabetes mellitus. Clinica Chimica Acta 365:230-5.

Wilson PWF, Garrison RJ, Abbott RD, Castelli WP. (1983). Factors associated with lipoprotein cholesterol levels. Arteriosclerosis 3:273-81.

Yambe T, Yoshizawa M, Saijo Y, Yamaguchi T, Shibata M, Konno S, Nitta S, Kuwayama T. (2004). Brachio-ankle pulse wave velocity and cardio-ankle vascular index (CAVI). Biomed Pharmacother 58:S95-8. 
Table 1. Characteristics of the study participants.

\begin{tabular}{|c|c|c|c|}
\hline & $\operatorname{Men}(n=1165)$ & Women $(\mathrm{n}=2573)$ & All $(n=3738)$ \\
\hline Age (y) & $66.0 \pm 10.9$ & $64.0 \pm 11.2 *$ & $64.6 \pm 11.2$ \\
\hline BMI $\left(\mathrm{kg} / \mathrm{m}^{2}\right)$ & $23.5 \pm 3.2$ & $22.9 \pm 3.4^{*}$ & $23.1 \pm 3.4$ \\
\hline $\mathrm{WC}(\mathrm{cm})$ & $85.0 \pm 8.7$ & $81.2 \pm 9.9^{*}$ & $82.4 \pm 9.7$ \\
\hline MBP (mmHg) & $103.0 \pm 12.9$ & $102.1 \pm 13.1 *$ & $102.7 \pm 13.1$ \\
\hline WBC $\left(10^{9}\right.$ cells $\left./ 1\right)$ & $6.0 \pm 1.5$ & $5.7 \pm 1.4^{*}$ & $5.8 \pm 1.4$ \\
\hline $\mathrm{RBC}\left(10^{10}\right.$ cells/1 $)$ & $457.3 \pm 43.9$ & $424.8 \pm 36.8^{*}$ & $434.9 \pm 41.9$ \\
\hline HGB (g/l) & $143.3 \pm 12.9$ & $128.6 \pm 11.3^{*}$ & $133.0 \pm 13.6$ \\
\hline $\mathrm{HCT}(\%)$ & $43.9 \pm 3.8$ & $40.0 \pm 3.3^{*}$ & $41.2 \pm 3.9$ \\
\hline CRE (mg/l) & $9.2 \pm 2.6$ & $7.1 \pm 1.7^{*}$ & $7.8 \pm 2.3$ \\
\hline $\mathrm{UA}(\mathrm{g} / \mathrm{l})$ & $61.5 \pm 13.9$ & $48.7 \pm 12.2^{*}$ & $52.7 \pm 14.1$ \\
\hline $\mathrm{TC}(\mathrm{g} / \mathrm{l})$ & $1.9 \pm 0.3$ & $2.1 \pm 0.3 *$ & $2.1 \pm 0.3$ \\
\hline TG $(g / 1)$ & $1.1(0.75-1.6)$ & $1.0(0.73-1.5)^{*}$ & $1.0(0.74-1.5)$ \\
\hline HDL-C (g/l) & $0.55 \pm 0.15$ & $0.62 \pm 0.15^{*}$ & $0.59 \pm 0.15$ \\
\hline LDL-C (g/l) & $1.1 \pm 0.3$ & $1.3 \pm 0.3 *$ & $1.2 \pm 0.3$ \\
\hline $\mathrm{HbA}_{1 \mathrm{c}}(\%)$ & $5.3 \pm 0.6$ & $5.2 \pm 0.6$ & $5.2 \pm 0.6$ \\
\hline CAVI & $8.5 \pm 1.6$ & $8.1 \pm 1.4^{*}$ & $8.2 \pm 1.5$ \\
\hline CIMT (mm) & $0.73(0.64-0.82)$ & $0.69(0.61-0.78)^{*}$ & $0.70(0.62-0.80)$ \\
\hline Current smoker & $264(22.7 \%)$ & $86(3.3 \%)^{*}$ & $350(9.4 \%)$ \\
\hline Past smoker & $445(38.2 \%)$ & $48(1.9 \%)^{*}$ & $493(13.2 \%)$ \\
\hline HT & $337(29.0 \%)$ & $704(27.4 \%)$ & $1041(27.8 \%)$ \\
\hline DM & $69(5.9 \%)$ & $88(3.4 \%)^{*}$ & $157(4.2 \%)$ \\
\hline DL & $62(5.3 \%)$ & $263(10.2 \%)^{*}$ & $325(8.7 \%)$ \\
\hline
\end{tabular}

Values are means \pm standard deviation or median $\left(25^{\text {th }}-75^{\text {th }}\right.$ percentile $)$.

BMI, body mass index; WC, waist circumference; MBP, mean blood pressure; WBC, white blood cells; RBC, red blood cells; HGB, hemoglobin; HCT, hematocrit; CRE, creatinine; UA, uric acid; TC, total cholesterol; TG, triglyceride; HDL-C, high-density lipoprotein cholesterol; LDL-C low-density lipoprotein cholesterol; $\mathrm{HbA}_{1 \mathrm{c}}$, hemoglobin $\mathrm{A}_{1 c}$; CAVI, cardio-ankle vascular index; CIMT, carotid intima-media thickness; HT, under treatment for hypertension; DM, under treatment for diabetes mellitus; DL, under treatment for dyslipidemia.

$* \mathrm{p}<0.01$ vs. men. 
Table 2. Univariate linear regression analysis of WBC and other variables.

\begin{tabular}{lllllll}
\hline Variables & Men & & Women & & All & \\
\hline Age & Correlation & P-value & Correlation & P-value & Correlation & P-value \\
BMI & $0.15^{\dagger}$ & $<0.001$ & $-0.076^{\dagger}$ & $<0.001$ & $-0.092^{\dagger}$ & $<0.001$ \\
WC & $0.18^{\dagger}$ & $<0.001$ & $0.11^{\dagger}$ & $<0.001$ & $0.14^{\dagger}$ & $<0.001$ \\
Smoking status & $0.2^{\dagger}$ & $<0.001$ & $0.1^{\dagger}$ & $<0.001$ & $0.14^{\dagger}$ & $<0.001$ \\
MBP & $0.272^{\dagger}$ & $<0.001$ & -0.016 & 0.41 & $0.2^{\dagger}$ & $<0.001$ \\
RBC & $0.1^{\dagger}$ & 0.001 & $0.043^{*}$ & 0.031 & $0.069^{\dagger}$ & $<0.001$ \\
HGB & $0.19^{\dagger}$ & $<0.001$ & $0.16^{\dagger}$ & $<0.001$ & $0.19^{\dagger}$ & $<0.001$ \\
HCT & $0.21^{\dagger}$ & $<0.001$ & $0.13^{\dagger}$ & $<0.001$ & $0.18^{\dagger}$ & $<0.001$ \\
CRE & $0.21^{\dagger}$ & $<0.001$ & $0.15^{\dagger}$ & $<0.001$ & $0.19^{\dagger}$ & $<0.001$ \\
UA & 0.034 & 0.25 & $0.078^{\dagger}$ & $<0.001$ & $0.092^{\dagger}$ & $<0.001$ \\
TC & $0.095^{\dagger}$ & 0.001 & $0.13^{\dagger}$ & $<0.001$ & $0.14^{\dagger}$ & $<0.001$ \\
logTG & $0.096^{\dagger}$ & 0.003 & $0.053^{\dagger}$ & 0.007 & $0.035^{*}$ & 0.046 \\
HDL-C & $0.26^{\dagger}$ & $<0.001$ & $0.2^{\dagger}$ & $<0.001$ & $0.22^{\dagger}$ & $<0.001$ \\
LDL-C & $-0.13^{\dagger}$ & $<0.001$ & $-0.094^{\dagger}$ & $<0.001$ & $-0.12^{\dagger}$ & $<0.001$ \\
HbA & $0.079^{\dagger}$ & 0.007 & $0.053^{\dagger}$ & 0.008 & $0.044^{\dagger}$ & 0.007 \\
CAVI & $0.072^{*}$ & 0.014 & $0.053^{\dagger}$ & 0.007 & $0.062^{\dagger}$ & $<0.001$ \\
CIMT & 0.001 & 0.98 & -0.019 & 0.33 & 0.003 & 0.868 \\
\hline p & -0.009 & 0.77 & -0.018 & 0.37 & -0.002 & 0.916 \\
\end{tabular}

${ }^{*} \mathrm{p}<0.05 ;{ }^{\dagger} \mathrm{p}<0.01$. 
Table 3. Multivariate linear regression analysis of WBC adjusted for age.

\begin{tabular}{|c|c|c|c|c|c|c|c|c|c|}
\hline \multirow[t]{2}{*}{ Variables } & \multicolumn{3}{|l|}{ Men } & \multicolumn{3}{|c|}{ Women } & \multicolumn{3}{|l|}{ All } \\
\hline & $\beta$ & $95 \% \mathrm{CI}$ & P-value & $\beta$ & $95 \% \mathrm{CI}$ & P-value & $\beta$ & $95 \% \mathrm{CI}$ & P-value \\
\hline BMI & 0.77 & $0.49,1.0$ & $<0.001$ & 0.48 & $0.32,0.63$ & $<0.001$ & 0.6 & $0.47,0.73$ & $<0.001$ \\
\hline WC & 0.33 & $0.23,0.43$ & $<0.001$ & 0.17 & $0.12,0.23$ & $<0.001$ & 0.24 & $0.19,0.28$ & $<0.001$ \\
\hline Smoking status & 5.1 & $4.0,6.2$ & $<0.001$ & 0.0 & $-0.001,0.0$ & 0.32 & 4.3 & $3.6,5.0$ & $<0.001$ \\
\hline MBP & 0.12 & $0.057,0.19$ & $<0.001$ & 0.077 & $0.035,0.12$ & $<0.001$ & 0.1 & $0.067,0.14$ & $<0.001$ \\
\hline $\mathrm{RBC}$ & 0.054 & $0.033,0.075$ & $<0.001$ & 0.055 & $0.041,0.07$ & $<0.001$ & 0.061 & $0.05,0.072$ & $<0.001$ \\
\hline HGB & 2.1 & $1.4,2.8$ & $<0.001$ & 1.5 & $1.0,2.0$ & $<0.001$ & 1.8 & $1.5,2.2$ & $<0.001$ \\
\hline $\mathrm{HCT}$ & 0.72 & $0.48,0.96$ & $<0.001$ & 0.59 & $0.43,0.75$ & $<0.001$ & 0.68 & $0.57,0.8$ & $<0.001$ \\
\hline CRE & 4.0 & $0.59,7.4$ & 0.022 & 7.1 & $4.0,10.2$ & $<0.001$ & 0.7 & $5.0,9.1$ & $<0.001$ \\
\hline UA & 1.0 & $0.39,1.6$ & 0.002 & 1.6 & $1.1,2.0$ & $<0.001$ & 1.6 & $1.3,1.9$ & $<0.001$ \\
\hline $\mathrm{TC}$ & 0.031 & $0.003,0.059$ & 0.032 & 0.023 & $0.007,0.38$ & 0.004 & 0.013 & $-0.001,0.027$ & 0.059 \\
\hline $\log \mathrm{TG}$ & 15.4 & $11.8,19.0$ & $<0.001$ & 13.2 & $10.9,15.6$ & $<0.001$ & 14.3 & $12.4,16.3$ & $<0.001$ \\
\hline HDL-C & -0.15 & $-0.21,-0.087$ & $<0.001$ & -0.099 & $-0.13,-0.064$ & $<0.001$ & -0.13 & $-0.16,-0.1$ & $<0.001$ \\
\hline LDL-C & 0.032 & $0.002,0.061$ & 0.036 & 0.026 & $0.009,0.043$ & 0.003 & 0.021 & $0.006,0.035$ & 0.006 \\
\hline $\mathrm{HbA}_{1 \mathrm{c}}$ & 2.2 & $0.82,3.6$ & 0.002 & 1.7 & $0.77,2.6$ & $<0.001$ & 1.9 & $1.2,2.7$ & $<0.001$ \\
\hline CAVI & 0.85 & $0.24,1.5$ & 0.007 & 0.32 & $-0.13,0.78$ & 0.17 & 0.68 & $0.32,1.0$ & $<0.001$ \\
\hline CIMT & 6.8 & $0.58,12.9$ & 0.032 & 2.7 & $-1.7,7.1$ & 0.23 & 5.4 & $1.9,9.0$ & 0.003 \\
\hline
\end{tabular}

$\beta$, standardized regression coefficient; CI, confidence interval. Other abbreviations are as shown in Table 1. 
Table 4. Multivariate linear regression analysis of WBC adjusted for confounding factors including CAVI.

\begin{tabular}{|c|c|c|c|c|c|c|c|c|c|}
\hline Variables & Men & & & Women & & & All & & \\
\hline & $\beta$ & $95 \% \mathrm{CI}$ & P-value & $\beta$ & $95 \% \mathrm{CI}$ & P-value & $\beta$ & $95 \% \mathrm{CI}$ & P-value \\
\hline Age & -0.19 & $-0.28,-0.1$ & $<0.001$ & -0.18 & $-0.23,-0.12$ & $<0.001$ & -0.18 & $-0.23,-0.13$ & $<0.001$ \\
\hline BMI & 0.64 & $0.35,0.93$ & $<0.001$ & 0.33 & $0.17,0.5$ & $<0.001$ & 0.42 & $0.27,0.56$ & $<0.001$ \\
\hline Smoking status & 5.1 & $4.0,6.2$ & $<0.001$ & -5.7 & $-0.001,0.001$ & 0.88 & 3.9 & $3.2,4.6$ & $<0.001$ \\
\hline MBP & 0.066 & $-0.002,0.13$ & 0.055 & 0.049 & $0.006,0.092$ & 0.025 & 0.059 & $0.023,0.096$ & 0.001 \\
\hline $\mathrm{HbA}_{1 \mathrm{c}}$ & 0.9 & $-0.45,2.3$ & 0.19 & 1.1 & $0.2,2.1$ & 0.017 & 1.2 & $0.4,1.9$ & 0.003 \\
\hline HDL-C & -0.093 & $-0.15,-0.034$ & 0.002 & -0.075 & $-0.11,-0.04$ & $<0.001$ & -0.077 & $-0.11,-0.047$ & $<0.001$ \\
\hline CAVI & 0.61 & $0.06,1.3$ & 0.043 & 0.35 & $-0.14,0.78$ & 0.17 & 0.4 & $0.044,0.76$ & 0.028 \\
\hline
\end{tabular}

$\beta$, standardized regression coefficient; CI, confidence interval. Other abbreviations are as shown in Table 1. 
Table 5. Multivariate linear regression analysis of WBC adjusted for confounding factors including CIMT.

\begin{tabular}{|c|c|c|c|c|c|c|c|c|c|}
\hline Variables & Men & & & Women & & & All & & \\
\hline & $\beta$ & $95 \% \mathrm{CI}$ & P-value & $\beta$ & $95 \% \mathrm{CI}$ & P-value & $\beta$ & $95 \% \mathrm{CI}$ & P-value \\
\hline Age & -0.17 & $-0.25,-0.078$ & $<0.001$ & -0.16 & $-0.22,-0.1$ & $<0.001$ & -0.16 & $-0.21,-0.12$ & $<0.001$ \\
\hline BMI & 0.62 & $0.33,0.91$ & $<0.001$ & 0.32 & $0.15,0.49$ & $<0.001$ & 0.4 & $0.26,0.55$ & $<0.001$ \\
\hline Smoking status & 5.1 & $4.0,6.2$ & $<0.001$ & -6.9 & $-0.001,0.001$ & 0.85 & 3.9 & $3.2,4.6$ & $<0.001$ \\
\hline MBP & 0.072 & $0.005,0.14$ & 0.036 & 0.052 & $0.008,0.095$ & 0.019 & 0.063 & $0.027,0.099$ & 0.001 \\
\hline $\mathrm{HbA}_{1 \mathrm{c}}$ & 1.0 & $-0.35,2.4$ & 0.15 & 1.2 & $0.25,2.1$ & 0.013 & 1.2 & $0.46,2.0$ & 0.002 \\
\hline HDL-C & -0.094 & $-0.15,-0.034$ & 0.002 & -0.075 & $-0.11,-0.04$ & $<0.001$ & -0.078 & $-0.11,-0.048$ & $<0.001$ \\
\hline CIMT & 2.5 & $0.06,1.3$ & 0.41 & 0.84 & $-0.14,0.78$ & 0.71 & 1.2 & $0.044,0.76$ & 0.51 \\
\hline
\end{tabular}

$\beta$, standardized regression coefficient; CI, confidence interval. Other abbreviations are as shown in Table 1. 